\title{
Spectroscopic study of the pulsations in the roAp star HD 24712
}

M. Sachkov ${ }^{1}$, T. Ryabchikova ${ }^{1,2}$, S. Bagnulo ${ }^{3}$, I. Ilyin ${ }^{4}$, T. Kallinger ${ }^{2}$, O. Kochukhov ${ }^{5}$, F. Leone ${ }^{6}$, G. Lo Curto $^{3}$, T. Lüftinger ${ }^{2}$, D. Lyashko ${ }^{7}$, A. Magazzu ${ }^{8}$, L. Mashonkina ${ }^{1}$ \& W.W. Weiss ${ }^{2}$

\footnotetext{
${ }^{1}$ Institute of Astronomy, Russian Academy of Science, 48 Pyatnitskaya str., 119017 Moscow, Russia

${ }^{2}$ Institut für Astronomie, Türkenschanzstrasse 17, 1180 Vienna, Austria

${ }^{3}$ European Southern Observatory, Casilla 19001, Santiago 19, Chile

${ }^{4}$ Astrothysikalisches Institut Potsdam, An der Sternwarte 16,D-14482 Potsdam, Germany

${ }^{5}$ Department of Astronomy and Space Physics, Uppsala University, Box 515, SE-751 20 Uppsala, Sweden

${ }^{6}$ INAF - Osservatorio Astrofisico di Catania, Via S. Sofia 78,95123 Catania, Italy

${ }^{7}$ Tavrian National University, Simferopol, Ukraine

${ }^{8}$ INAF - Telescopio Nazionale Galileo, PO Box 565, 38700 Santa Cruz de La Palma, Spain
}

\begin{abstract}
We present results of the radial velocity (RV) analysis of spectroscopic time-series observations of the roAp star HD 24712 (HR 1217). Only lines of the rare-earth elements (REE) show substantial amplitudes in the RV data. The progressive change of the RV pulsation phase with the optical depth for lines of the same element gives strong evidence for a running wave. The surface abundance distribution should be taking into account in interpreting of the phase shifts between the RV curves for different elements. We did not find phase jumps at the magnetic field extrema reported earlier by Mkrtichian \& Hatzes (2005).
\end{abstract}

\section{Spectroscopic observations}

We obtained time-series high-resolution spectroscopic observations. They were carried out at ESO during November 10/11, 2004 (HARPS - 92 spectra, 60 sec time resolution, $\mathrm{S} / \mathrm{N}=120$ ), November $11 / 12$ and $12 / 13$ (UVES - 92 \& 73 spectra, $50 \mathrm{sec}, \mathrm{S} / \mathrm{N}=300$ ) and 35 spectra were obtained on November 13/14, 2004 with SARG at TNG. The spectroscopy covers the rotation phases of $0.867,0.944,0.028$, and 0.176 near the magnetic and pulsation amplitude maximum according to the most recent ephemeris (Ryabchikova et al.2005). All spectra were reduced and normalized to the continuum level either with MIDAS and IRAF or with a routine specially developed by one of us (DL) for a fast reduction of time-series observations. We also used observations with Gecko coudé spectrograph at the 3.6-m Canada-France-Hawaii telescope in September 22-25 2002 (partially described by Sachkov et al. 2004) which were obtained near the magnetic and pulsation amplitude minimum.

\section{Radial velocity and pulsational phase shifts}

Radial velocities of more that 500 unblended spectral lines were measured. About $1 / 3$ of all pulsating lines could not be identified, but according to their pulsation characteristics they should belong to rare-earth elements (REE). Pulsational curves for different element convolved with the main period $(\mathrm{P}=6.125 \mathrm{~min})$ are presented in Fig. 1 . One clearly sees the 
growth of pulsation amplitude towards the upper atmosphere as well as progressive phase shifts. NLTE optical depths of line formation for Nd (Mashonkina, Ryabchikova \& Ryabtsev 2005) and for hydrogen lines are shown on Fig. 1. For the Ca lines, the depth formation was calculated based on an LTE stratification study.

Phase shifts for the lines of the same element may be explained by the running wave in a stratified atmosphere of the star. NLTE calculations of Nd lines' depth formation within an $\mathrm{Nd}$ overabundant layer confirms this (see Fig. 2). If phase shifts are caused by vertical stratification only, REE like Pr and Tb should be concentrated higher than Nd in the stellar atmosphere. But phase shifts between lines of different elements may partially be explained by different surface distributions of these elements. Not all REE concentrate in the same spot (see, for example, Pr, Nd and Eu maps in the roAp star HD 3831 - Kochukhov et al. 2004). A NLTE study of Pr and Tb depths formation and Zeeman Doppler imaging which are in progress are crucial for understanding pulsation in roAp stars.

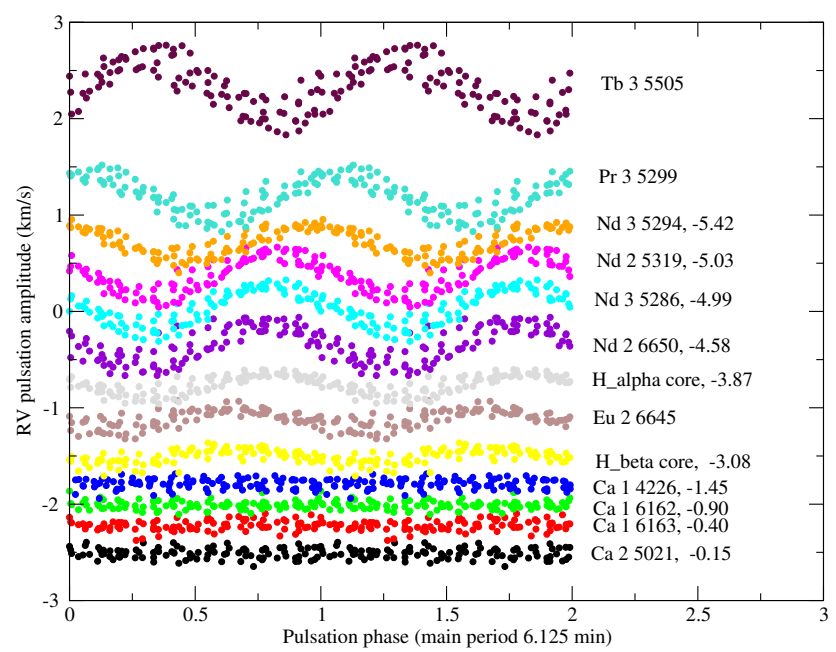

Figure 1: Phase shifts between RV variations in the lines of different elements/ions .

\section{Line pulsational behaviour before and after the Balmer Jump}

For the first time RV measurements were made in spectral regions before and after the Balmer Jump (BJ) using the UVES spectra. It allows to make an additional test for the pulsation distribution through the atmosphere, because spectral lines in a homogeneous atmosphere should be formed at different optical depth by definition. $\mathrm{Ca}, \mathrm{Mn}, \mathrm{Fe}, \mathrm{Co}, \mathrm{Ni}$ lines before and after the BJ do not show measurable RV amplitudes, while RV amplitudes and phases for REE elements before the BJ follow the same dependence on optical depth as the lines lying after the BJ. This supports an element stratification model in the atmospheres of roAp stars where REE are concentrated in the upper atmospheres and REE lines are formed in these upper layers independently on their wavelength position. No pulsations were found in the lines of the heavy elements Pd I and Rh II. 


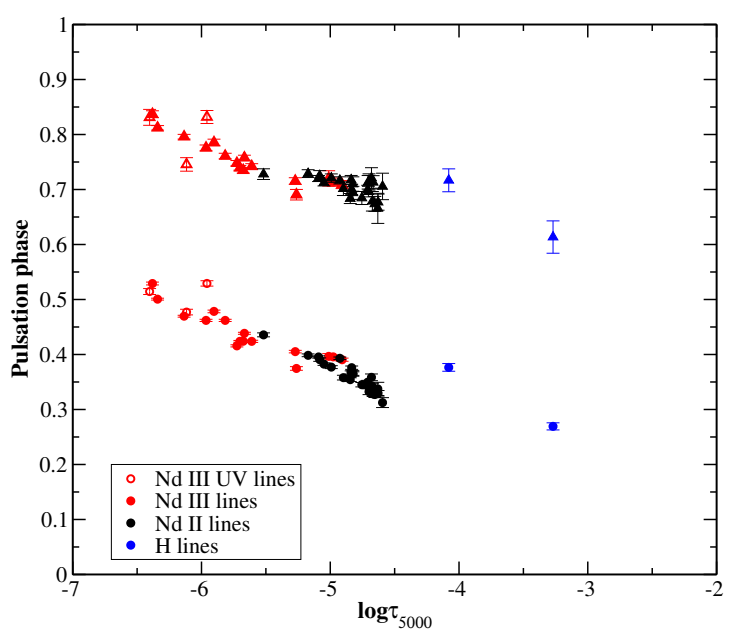

Figure 2: Phase of pulsation maximum vs. depths of line formation. Dots and triangles indicate phase shifts for two pulsation periods: $6.125 \mathrm{~min}$ (main frequency) and for $6.282 \mathrm{~min}$.

Table 1: Rotational phases (as a fraction of the rotation period) of the data sets and corresponding pulsation phases (as a fraction of the pulsation period) for Nd III

\begin{tabular}{|c|c|c|c|}
\hline \multicolumn{2}{|c|}{2002} & \multicolumn{2}{c|}{2004} \\
\hline Ph(rot) & Ph(puls) & Ph(rot) & Ph(puls) \\
\hline 0.284 & 0.84 & 0.867 & 0.52 \\
0.361 & 0.72 & 0.944 & 0.56 \\
0.443 & 0.76 & 0.028 & 0.61 \\
0.528 & 0.75 & 0.176 & 0.62 \\
\hline
\end{tabular}

\section{Pulsation phase jumps}

Recently Mkrtichian \& Hatzes (2005) found the pulsation phase " $\pi$ - switching" exactly at the magnetic field and pulsation amplitude extrema. This is inconsistent with the oblique pulsator model (Kurtz \& Shibahashi 1986). We have no continuous data set which covers the whole rotation period. But two observation sets - around magnetic maximum in 2004 and around magnetic minimum in 2002 - were performed in 4 consecutive nights each (see Table 1). We did not find any pulsation phase jumps within both observational sets. Because of the very small pulsation period (about $6 \mathrm{~min}$ ) it is very difficult to avoid false shifts phasing together observations separated by large time intervals. The phase jumps in the data of Mkrtichian \& Hatzes, which occur exactly between two data sets separated by a month (more than 9000 pulsation periods), are most probably caused for this reason.

Acknowledgments. This work was supported by the RFBR grant 04-02-16788, by the Presidium RAS program 'Non-stationary phenomena in astronomy', by the Austrian FFGALR (MOST Ground Station) and Science Fonds (FWF-P17580N2). MS also gratefully acknowledges the support of European Astronomical Society. 


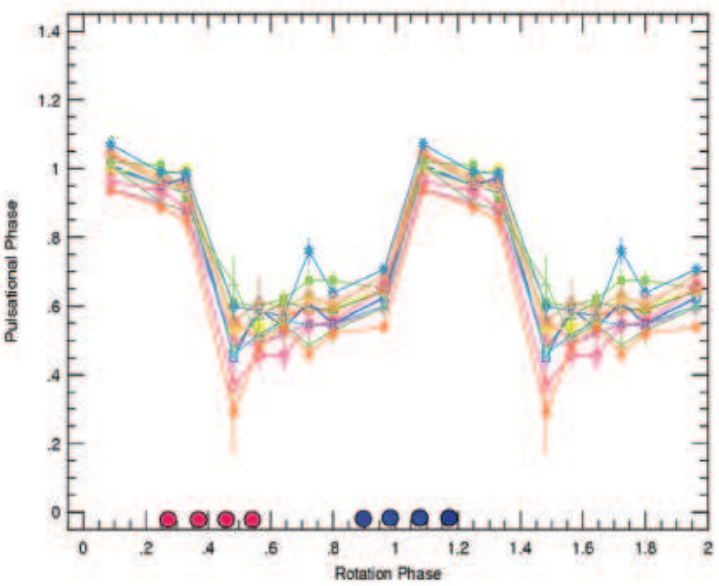

Figure 3: Pulsation phase jump (from Mkrtichian \& Hatzes, 2005). Circles at the bottom show rotation phases of our data sets.

\section{References}

Kochukhov, O., et al. 2004, A\&A 424, 935

Kurtz, D.W., Shibahashi, H. 1986, MNRAS 223, 557

Mashonkina, L. I., Ryabchikova, T.A., Ryabtsev, A.N. 2005, A\&A 441, 309

Mkrtichian, D.E., Hatzes, A.P. 2005, A\&A 430, 263

Ryabchikova, T., et al. 2005, A\&A 429, L55

Sachkov, M., et al. 2004, in "Variable Stars in the Local Group", eds. D.W. Kurtz, K.R. Pollard, ASP Conf. Ser. 310, 208 